\title{
The First-Order Derivative of Atmospheric Electronic Field in an Optimal Time Window Improves the Accuracy of Lightning Warning
}

\author{
LUO Fei ${ }^{1, a}$, FENG Shuai ${ }^{1, b}$, BIAN Yajing ${ }^{1, c}$ and LIU Juan ${ }^{1, d, *}$ \\ ${ }^{1}$ Wuhan University, Computer School, Wuhan, Hubei, China. \\ aluofei_whu@126.com, bfs2003@126.com, cwhucs_bianyj@whu.edu.cn, diujuan@126.com
}

Keywords: lightning warning, atmosphere electronic field.

\begin{abstract}
Lightning is a major threat to people's daily activities. Therefore, to forecast its occurrence is very important to ensure the safety of life and property. Compared to a series of signals from instruments like the Doppler radar, the lightning locator and the meteorological satellite, the electromagnetic field signal is easy to be detected by the atmosphere electronic field instrument and strongly correlated with lightning. We analyze the defections of traditional warning methods through setting threshold on the atmospheric electronic field value and propose a new method which uses the first-order derivative to model the persistent and low-intensive fluctuation characteristic of atmospheric electronic field before the lightning. The experiment on real data proves that our method could improve the lightning warning accuracy.
\end{abstract}

\section{Introduction}

Lightning is a kind of meteorological phenomenon. It occurs during an electrical storm between electrically charged regions of a cloud (called intra-cloud lightning), between that cloud and another cloud (Cloud to Cloud lightning), or between a cloud and the ground (Cloud to Ground lightning). Lightning could discharge strong electromagnetic radiation and large current. For example, in a short time, the current could reach up to $300 \mathrm{kA}$. Meanwhile, there are heating effect and complex electromagnetic waves. Therefore, lightning is a major threat to people's daily activities and infrastructures such as oil stocks, energy pipelines, telecommunication and traffic transportation.
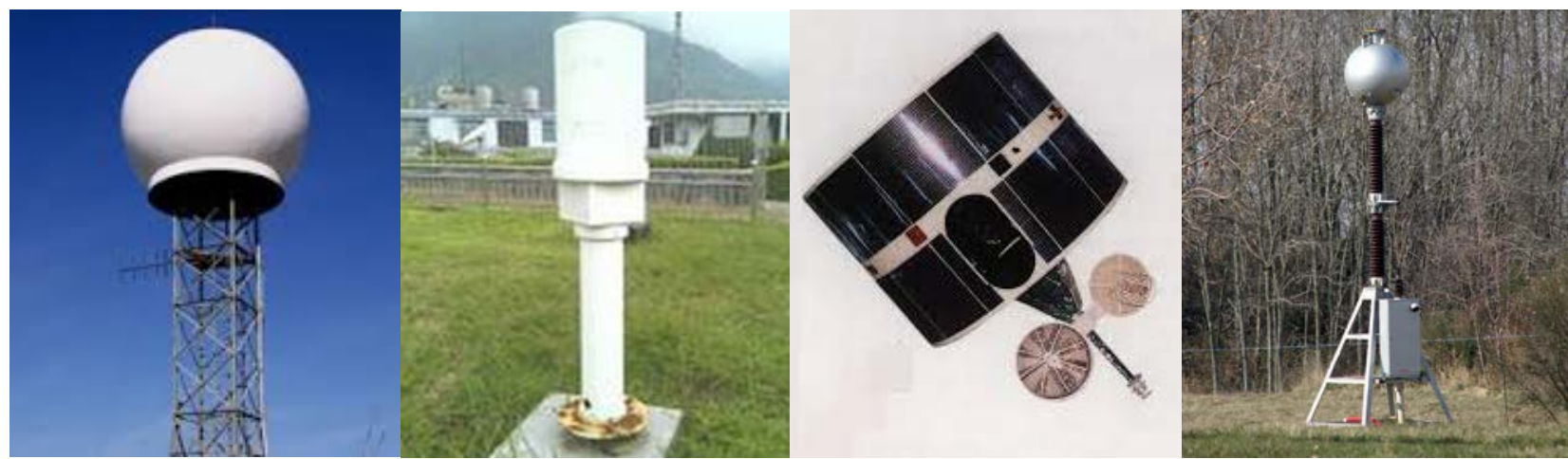

Fig.1. Common instruments to detect lightning. From left to right is the Doppler radar, lightning locator, meteorological satellite and atmosphere electronic field instrument.

A series of instruments are invented to detect the signals from the lightning as shown in the figure 1. Doppler radar sends directional pulses of microwave radiation. When the microwave travels in the air, the precipitations could absorb and scatter the microwave with different ratio. Through analyzing the radar echo, it's able to locate the precipitation, calculate its motion, and estimate whether it is rain, snow, hail or others [1]. Some of them are necessary components of the lightning. Another instrument is the lightning locator. It makes use of electromagnetic characteristic of lightning to remotely sense lightning [2, 3]. Meteorological satellite could send the images of the earth to the control and analysis center on the ground. Processing the images, the lightning could be identified [4]. Thunderstorm generator hypothesis indicates that charged particles are transferred from the bottom of thunder 
clouds to the surface of the Earth and from the top of the thunder clouds to the ionosphere. Therefore, there is a close correspondence between the variations of the electrical potential gradient and the variation of thunderstorm and electrical showers clouds occurrence [5, 6]. Based on such relationship, the atmosphere electronic field instruments could be placed over a large area and organized in a net to monitor lightning's activity. It is a cheap and real-time way to monitor the lightning [7]. Here, we focus on using the atmosphere electronic field data to warn the lightning hazard. Besides the hardware's supporting, methods to judge lightning from the raw data is necessary. So far, the warning methods could be categorized into the following types. The first is called the fixed threshold method. When the atmosphere electronic field value climbs over certain threshold, one lightning is thought to take place [8]. Because this fixed threshold is hard to identify, the second type called the grading method uses multiple atmosphere electronic field values to rank multiple lightning hazard levels [9]. The third type called auto-adapted threshold tries to find a proper threshold with the help of some intelligent algorithms [10]. Above all methods directly or indirectly need to decide the important atmosphere electronic field threshold. But for different regions, criterion could be different. The most important problem is that atmosphere electronic field is not only related to lightning, other meteorological factors also affect its value. For example, in a sunshine day, strong wind, fog and haze, and sand storm can also increase the atmosphere electronic field value. Therefore, this work proposes a new more reliable lightning hazard warning method to solve this problem.

\section{Method}
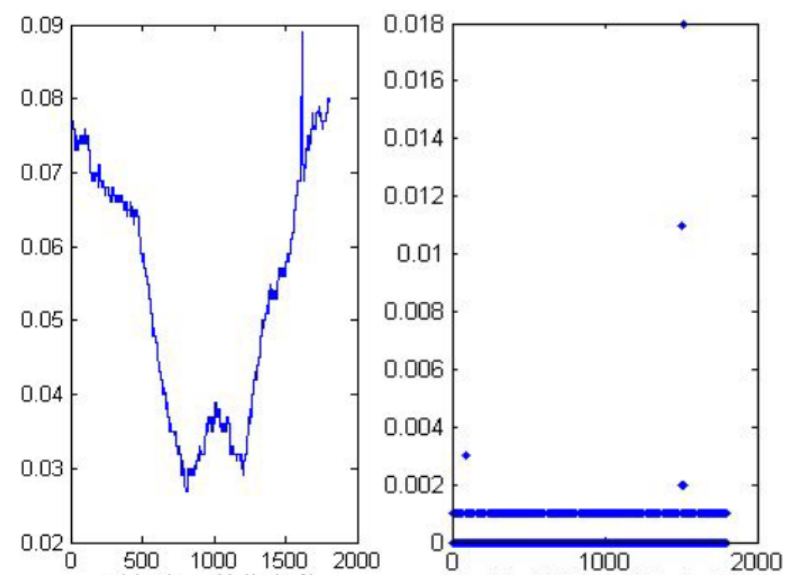

(a)
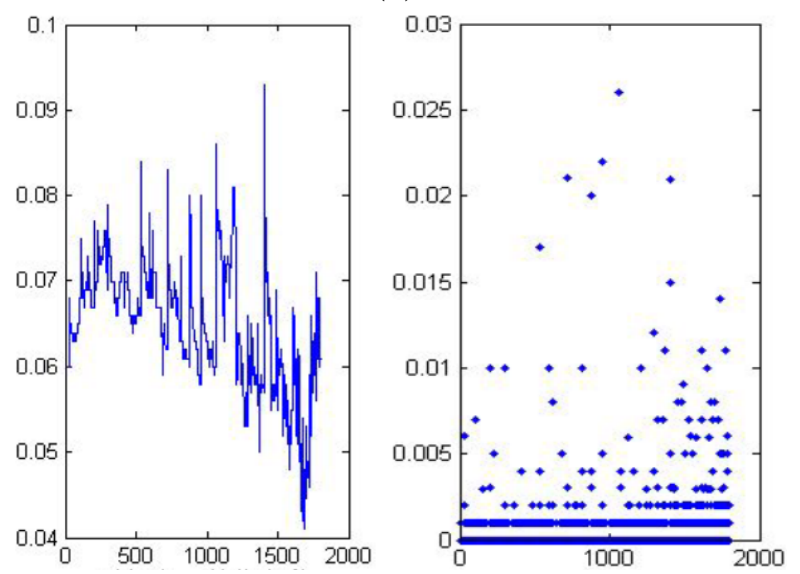

(b)

Fig. 2. Atmosphere electronic field value curves under different whether situations. (a) Sunshine day. The left is the raw atmosphere electronic field values on consecutive 2000 time points. The right is the first-order derivative of left part data. (b) Lightning day. The left is the raw atmosphere electronic field curve on consecutive 2000 time points for a lightning. The right is the first-order derivative of left part data. 
Many real monitoring data shows that it's unreasonable to do lightning warning with the absolute magnitude of atmosphere electronic field. Figure 2 is a negative case that in the sunshine and non-lightning day, the atmosphere electronic field can reach very high value. Hence, high atmosphere electronic field threshold couldn't ensure the accuracy of threshold methods.

We investigate the characteristics of atmosphere electronic field under lightning days and sunshine days and find that a typical lightning process is not a sudden event. It contains several stages, including forming, lasting discharging, and ending stage as shown in the figure 3.

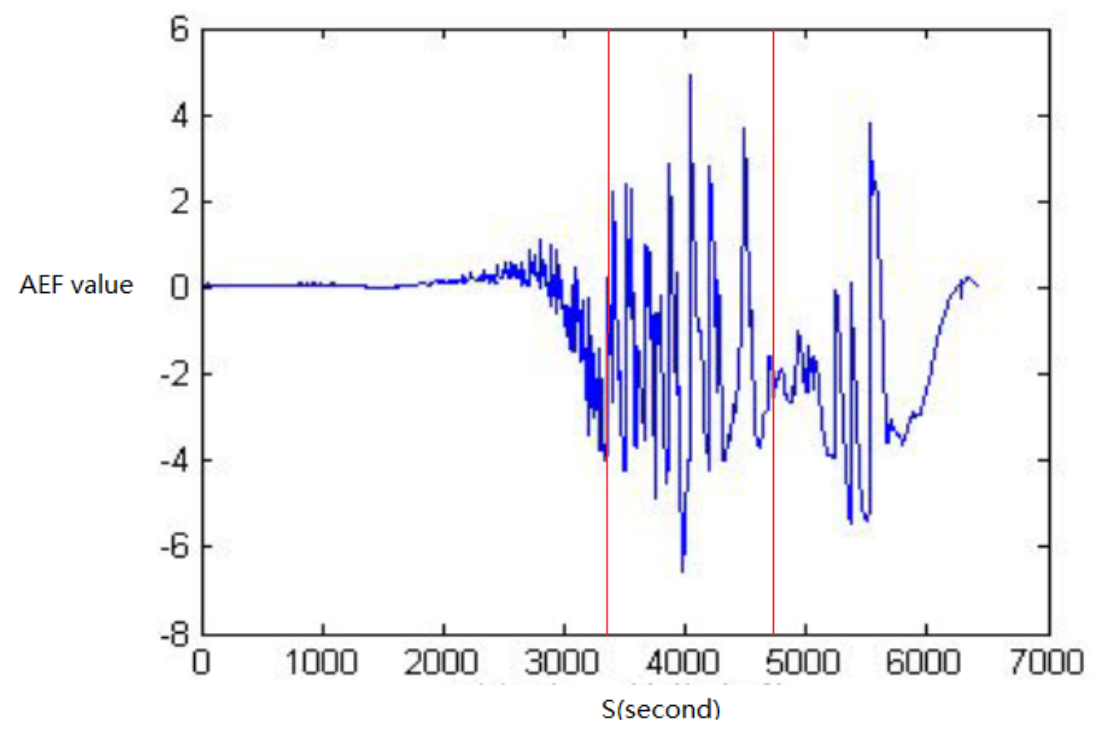

Fig. 3, atmosphere electronic field value of a typical lightning process. The two vertical lines separate three stages. From left to right sections are forming, lasting discharging and ending.

Each stage has remarkable differences at the aspects of atmosphere electronic field strength, fluctuation frequency and polarity. As far as the forming stage concerned, before the large-scale and high-intensive lightning stage arrives, the atmosphere electronic field has many low-intensive fluctuations and the total trend gradually increases. Compared it with the high value caused by other factors rather than lightning, the curve in the figure 2 (a) left part is smoother. Therefore this important difference could be used to distinguish the true positive and false positive lightning. We use the first order of derivative of atmosphere electronic field to formulate lightning forming stage's low-intensive fluctuation. Because the instrument outputs discrete results, we use the approximate version to calculate the derivative.

$$
f^{\prime}=\left(f\left(t_{i}\right)-f\left(t_{j}\right)\right) /\left(t_{\mathrm{i}}-t_{\mathrm{j}}\right) .
$$

where the $f(t)$ is the atmosphere electronic field output function at the time point $t$. The $t_{\mathrm{i}}-t_{\mathrm{j}}$ is the minimum interval between the neighboring outputs, which is determined by the instrument and usually equals to one second. After getting the derivative, we could get the distribution shown in the right part of figure 2 (a) and (b). Meanwhile, in order to emphasize the fluctuations' persistence, we need to calculate the amount of effective derivative whose value exceeds certain threshold. Notable, this derivative threshold is the changing rate rather other absolute value of atmosphere electronic field. Thus it is more stable for different meteorological and geographic conditions. A two-dimension parameter $(n, l)$ denotes the number $n$ of effective derivative falling in the time interval of $l$. We use the history data of the focused region and GA algorithm to optimize one ( $\left.n^{\prime}, l^{\prime}\right)$, which could perfectly classify the lightning appearance. This ( $\left.n^{\prime}, l^{\prime}\right)$ would be used as criterion in the future lightning warning for this focused region. 


\section{Experiment}

In order to test our method's performance, we make an experiment on an atmosphere electronic field data from August, 2013 to December, 2013 in Jiangyin city and Yixing city, Jiangsu province, China. The experiment randomly extracts 30 lightning occurrences. The time interval of each atmosphere electronic field record data covers two hours before lightning. And then, also randomly pick out 30 samples in the sunshine days as the negative samples. Table 1 is the result of our method.

Table 1. The prediction contingency table

\begin{tabular}{|c|c|c|c|c|}
\hline & \multicolumn{3}{|c|}{ Observed } \\
\hline & & yes & no & total \\
\hline \multirow[t]{3}{*}{ Forecast } & yes & 24 & 3 & 27 \\
\hline & no & 6 & 27 & 33 \\
\hline & total & 30 & 30 & 60 \\
\hline
\end{tabular}

With above basic results that $\mathbf{E A}=24$ (Effective Alarm, observed lighting that is correctly predicted), FA $=3$ (The False Alarm, lightning warning triggered, but none lightning stroke observed) and FTW=6 (The Failure to Warn, lightning observed without a previous triggered warning), Three categorical statistics of the probability of detection, the false alarm ratio and Ts score assess the effectiveness and efficiency of the prediction.

$$
\begin{array}{r}
\text { POD }=\mathbf{E A} /(\mathbf{E A}+\mathbf{F T W})=24 /(24+6)=0.80 \\
\text { FAR }=\mathbf{F A} /(\mathbf{F A}+\mathbf{E A})=3 /(3+24)=0.11 \\
\text { Ts }=\mathbf{E A} /(\mathbf{E A}+\mathbf{F A}+\mathbf{F T W})=24 /(24+6+3)=0.73
\end{array}
$$

In order to prove that our method is superior to the traditional threshold method, we compare two methods' performances. Because the most suitable threshold is unknown for traditional method, we assume that a range of thresholds could cover the best threshold and use the best prediction result to compare two methods. The step of neighboring thresholds is decided by our atmosphere electronic field instrument's minimum detection precision, which is 0.001 .

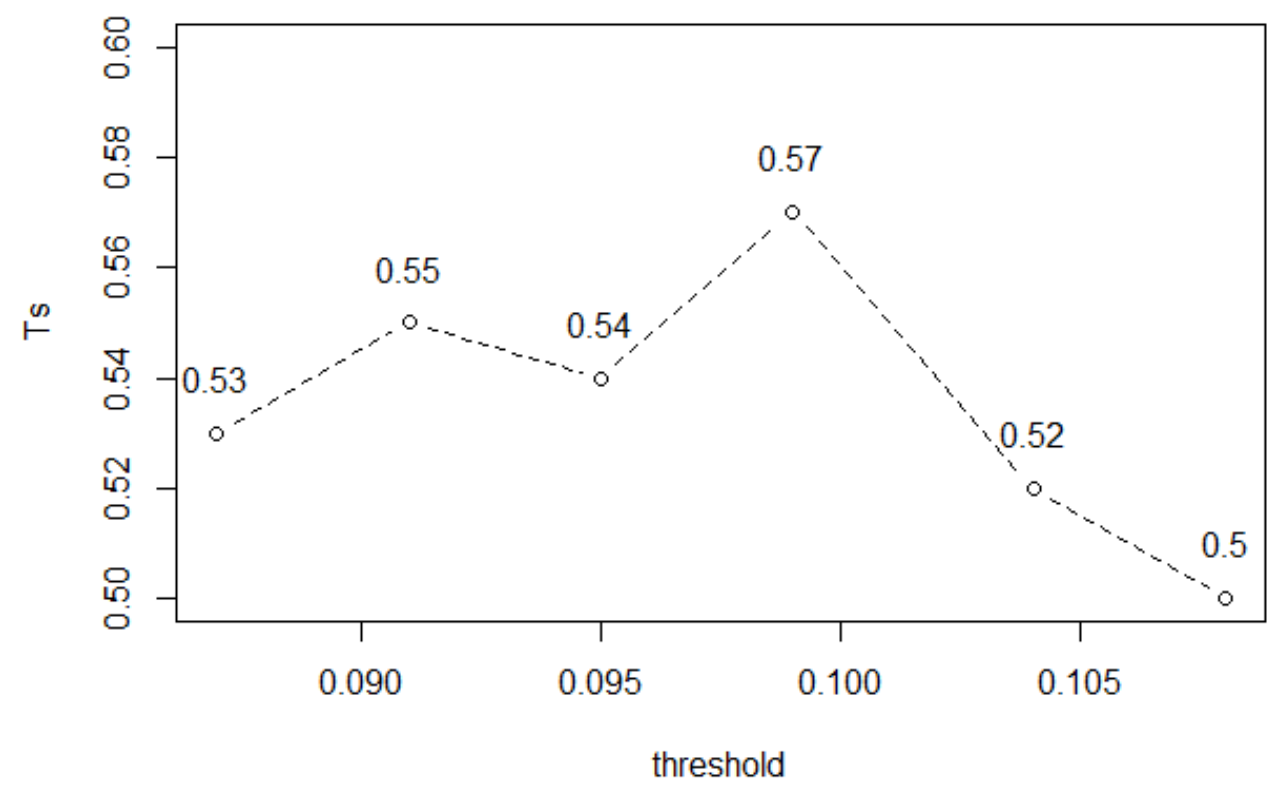

Fig. 4 Ts results on thresholds. 
The figure 4 depicts the Ts value curve on a range of thresholds for traditional method. Ts climbs with the threshold value increasing, reaches the maximum value 0.57 around 0.099 and decreases quickly after that. Table 2 lists the detailed comparison results.

Table 2. Comparison of traditional method and our method

\begin{tabular}{ccc}
\hline Evaluation Index & Threshold method & Our method \\
\hline EA & 19 & 24 \\
FA & 3 & 3 \\
FTW & 11 & 6 \\
Ts score & 0.57 & 0.73 \\
FA ratio & 0.14 & 0.11 \\
FTW ratio & 0.37 & 0.20 \\
\hline
\end{tabular}

\section{Summary}

In this work, we summarize the principles of traditional instruments for lightning warning, such as radar, lightning locators and satellites, and points out their weaknesses. In order to improve the warning accuracy, we propose a new method based on the atmospheric electric field data. Use the first-order derivative during a time window before the arrival of great lightning to model the key feature for lightning occurrence. By comparing it with the traditional method, our 0.73 Ts score is much higher than 0.57 of the traditional method. Thus our method could be applied in real lightning warning.

\section{Acknowledgement}

This work is supported by the National Science Foundation of China [61402340, 61572368], the program for new Century Excellent Talents in Universities [NCET-10-0644] and Natural Science Foundation of Hubei Province of China [2014CFB194].

\section{References}

[1] Yue C, Desheng C, Zaihua G. Research on the Relationship of Doppler Radar and the Lightning Warning [J]. Meteorological and Environmental Sciences, 2010, 1: 006.

[2] Zifu, L, Yuehong Y, Xiaoli S, et al. The Potential Prediction of Thunderstorm and Monitoring, Nowcasting and Warning System in Jiaozuo, Henan Province. Meteorological Monthly 8 (2007): 018.

[3] Cummins K L, Murphy M J. An Overview of Lightning Locating Systems: History, Techniques, and Data Uses, With an In-Depth Look at the U.S. NLDN. IEEE Transactions on Electromagnetic Compatibility, 2009, 51(3):499 - 518.

[4] Zhou Y, Tian M, Zhang Q L, et al. Design and implementation of lightning identification system on meteorological satellite. Optics and Precision Engineering, 2012, 7: 026.

[5] Gołkowski M, Kubicki M, Cohen M, et al. Estimation of global lightning activity and observations of atmospheric electric field. Acta Geophysica, 2011, 59(1): 183-204..

[6] Raulin J P, Tacza J, Macotela E, et al. A New South America Electric Field Monitor Network. Sun and Geosphere, 2014, 9: 111-114.

[7] Kosmac J, Djurica V, Babuder M. Automatic fault localization based on lightning information. IEEE Power Engineering Society General Meeting, 2006

[8] Chai R, et al. Application of Atmospheric Electric Field Data in Lightning Warning. Meteorological Science and Technology, Vol.37, No.6 (2009). pp: 724-728. 
[9] Lynn B H, Yair Y, Price C, et al. predicting cloud-to-ground and intracloud lightning in weather forecast models. Weather and Forecasting, 2012, 27(6): 1470-1488.

[10] Guo Juntian, et al. A dynamic adaptive threshold method to forecast the lightning based on the atmospheric electric field. The 30th meteorological society of China annual meeting. 2013 